\title{
Symbolic Dynamics, Entropy and Complexity of the Feigenbaum Map at the Accumulation Point
}

\author{
WERNER EBELING ${ }^{\mathrm{a}, *}$ and KATJA RATEITSCHAK ${ }^{\mathrm{b}}$ \\ a Institute of Physics, Humboldt University Berlin, Invaliden Str. 110, D-10115 Berlin, Germany; \\ ${ }^{\mathrm{b}}$ Center for Nonlinear Phenomena and Complex Systems, Free University of Brussels, B-1050 Brussels, Belgium
}

(Received 14 February 1998)

\begin{abstract}
This paper aims to make further contributions to the exploration of the symbolic dynamics generated by the logistic map at Feigenbaum accumulation point. In particular we are interested in the grammar of these sequences; completing earlier studies we study here arbitrary partitions also. Our main aim is the investigation of the special grammars which characterize the long-range correlations between letters. Considering these sequences as standard examples of a complex system, we introduce and discuss a complexity function derived from the conditional entropies. Further we discuss local predictabilities.
\end{abstract}

Keywords: Feigenbaum map, Shannon entropy, Complexity measures

\section{INTRODUCTION}

One of the best studied systems in nonlinear dynamics is the logistic map at Feigenbaum accumulation point, where multiperiodicity changes into chaos [1-3]. Feigenbaum investigated in detail the properties at this critical point of the dynamics and in particular the scaling behaviour [1]. In this article we would like to make further contributions to the exploration of the properties of the symbolic dynamics generated by the Feigenbaum map. We consider the Feigenbaum sequences as prototypes of complex systems, which are characterized by a hierarchy of structures and correlations on all scales. In particular we are interested in the grammar of these sequences, which appears to be a special but very powerful tool to characterize these structures. Completing earlier studies [4-7] we study here arbitrary partitions also.

As well known, the special grammar which characterizes the accumulation point and in particular the long-range correlations between letters are connected with the criticality of the dynamics. In nonlinear dynamics and in statistical mechanics criticality plays a special role. We know for example that thermodynamical systems at critical values of temperature and pressure have very special properties. In particular we know that critical conditions imply the existence of long-range correlations with respect to time and space having special scaling

* Corresponding author. 
properties. As shown first by Haken and Schlögl, nonequilibrium systems may behave in a quite similar way, if the control parameters are near to a bifurcation point. In particular we may expect that there exist long-range correlations and scaling properties. Several structures generated by evolutionary processes and in particular human writings seem to have similar properties [8].

As shown by Grassberger [3] and in our earlier work [4-6] the logistic map operating at the accumulation point shows infinite-range correlations of a very special type. The purpose of this paper is the investigation of these long-range correlations using methods of entropy and grammar analysis. Besides the principal interest in the analysis of long correlations our main aim is related here to the question of grammatical structures at the critical point. We consider the long-range correlations and hierarchical grammatical structures appearing in our particular example as prototypical for complex systems. Based on this view we propose in the last part a new measure of complexity which generalizes Grassberger's measure complexity and the classifications proposed by Szepfaluzy [13].

\section{FEIGENBAUM ACCUMULATION POINT, A GRAMMATICAL APPROACH}

The logistic map, defined by

$$
x_{n+1}=f\left(x_{n}\right)=r x_{n}\left(1-x_{n}\right),
$$

shows a lot of nonlinear phenomena, like period doubling scenario, intermittency, crises and fully developed chaos [2]. If one starts with the control parameter $r=1$ and increases $r$ then one can observe a route from order to chaos: the period doubling scenario. The transition to chaos occurs at the critical value $r_{\infty}=3.56994567 \ldots$, called Feigenbaum accumulation point. With decreasing distance to the accumulation point the complexity of the dynamics increases, proceeding from multiperiodicity to chaos. In this way the route to chaos in the logistic map may shed some light on the old problem as to what complexity is, how it arises and which measure can be applied to characterize it. The microscopic probability density of the Feigenbaum accumulation point has a Cantor structure [1]. Corresponding to the Cantor structure of the microscopic density the generating partition at $x_{\max }=\frac{1}{2}:\left[0, x_{\max }\right] \rightarrow 0$ and $\left(x_{\max }, 1\right] \rightarrow 1$ leads to a selfsimilar symbol sequence, which we want to call Feigenbaum sequence. Entropy analyses of the Feigenbaum sequence have been done by Grassberger [3] and ourselves [4,6,7].

A new approach in the investigation of the Feigenbaum sequence is based on a symbol sequence generator [6,7]. A symbol sequence generator is a set of deterministic and stochastic grammatical rules to construct symbol sequences.

If one applies the replacement rules

$$
\begin{aligned}
& 1 \rightarrow 10 \\
& 0 \rightarrow 11
\end{aligned}
$$

to an infinitely long Feigenbaum sequence then one gets the same symbol sequence. This rescaling invariance is the defining property of selfsimilarity. These replacement rules establish a link to grammatical rules in the context of formal languages [10]. We constructed a deterministic symbol sequence generator producing the binary Feigenbaum sequence on the basis of the replacement rules $[6,7]$ :

$$
\begin{aligned}
a_{0} & =1, \\
a_{1} & =10, \\
a_{n+1} & =a_{n} \circ a_{n-1} \circ a_{n-1} .
\end{aligned}
$$

Here $a_{i}$ denotes a symbol sequence of length $2^{i}$. The symbol $\circ$ means concatenation of two subsequences. The symbol sequence generator has the following property.

LEMMA 1 The sequences $a_{n}$ and $a_{n-1} a_{n-1}$ only differ in the last symbol.

One can find the proof of this lemma in [6].

Another example of a selfsimilar symbol sequence is the binary rabbit sequence. Investigations of this sequence including the calculation of 
the conditional entropies had been done by Gramss [11].

Selfsimilarity already occurs in the symbolic dynamics of the period doubling scenario. There are relations between our symbol sequence generator and a construction principle for the symbolic dynamics of the period doubling scenario introduced by Schroeder [12]. He constructed the symbol sequences of the superstable periodic orbits in the following way: One chose the partition: $x_{\max }=$ $\frac{1}{2} \rightarrow C,\left[0, x_{\max }\right) \rightarrow L$ and $\left(x_{\max }, 1\right] \rightarrow R$. A superstable orbit of the period $2^{k}$ is the starting point ((4), starting with $k=0$ ). First one writes two periods of the superstable orbit of the period $2^{k}$. If the number of $R$ left from the second $C$ is uneven, then the second $C$ has to be changed to $L$. Otherwise the second $C$ has to be changed to $R$ :

period 1: $C$

period $1 \rightarrow$ period 2: $C C \rightarrow C R$

period $2 \rightarrow$ period $4: C R C R \rightarrow C R L R$

period $4 \rightarrow$ period 8: CRLRCRLR

$\rightarrow C R L R R R L R$

This algorithm is connected with the microscopic dynamics. We consider $f(0.5, r)$ and $f(0.5, r+\delta)$. The attractor is the superstable periodic orbit of the period $2^{k}$ for the parameter $r$ and the superstable periodic orbit of the period $2^{k+1}$ for the parameter $r+\delta$. The gradient of the logistic map is negative in the interval $\left(x_{\max }, 1\right]$. If the microscopic iterates of these points fall into $R$ then a change of the sign in their distance $f^{i}(0.5, r+\delta)-f^{i}(0.5, r)$ occurs. An uneven number of sign changes leads to a sign change which means the second $C$ is changed to $L$. An even number of sign changes does not lead to a sign change which means the second $C$ is changed to $R$.

Our symbol sequence generator is obviously connected with the symbolic dynamics of the superstable periodic orbits. If one reads the $a_{i}$ in (3) in opposite direction then they correspond to the symbol sequences of the superstable periodic orbits in (4) until the last symbol (but the last symbol is not fixed in (4)). If one maps the $2^{k}$ th symbol of the period $2^{k+1}$ onto the first symbol of the period $2^{k}$ then one can read the sequence of period $2^{k+1}$ as a continuation of the sequence of period $2^{k}$ in opposite direction. The sequences constructed in (4) and read in opposite direction are identical to the sequences constructed in (3).

This connection also allows the conclusion that $x_{\max }=\frac{1}{2}$ is the microscopic starting point, which iterates exactly producing the symbol sequence generated by (3) on the macroscopic level.

\section{ARBITRARY PARTITIONS}

In the previous section we only considered symbol sequences resulting from the generating partition of state space. In this section we investigate symbol sequences resulting from arbitrary binary partitions of state space.

For this purpose we need our symbol sequence generator (3): On the basis of Lemma 1 one finds in the original binary Feigenbaum sequence repetitions of subsequences $A$ of length $2^{z}-1, z>0$ and there is one changing symbol, denoted $S_{0}, S_{1}$, $S_{2}, \ldots$, between two repetitions, Fig. 1. As a consequence of selfsimilarity the symbols $S_{0}, S_{1}$, $S_{2}, \ldots$ form the same selfsimilar sequence (besides a permutation of the alphabet).

The symbols $S_{i}$ have the same history and the same future. They correspond to a microscopic trajectory in the vicinity of 0.5 , either left of 0.5 (symbol 0 ) or right of 0.5 (symbol 1).

For all $m>0$ only one of the $2^{m}$ backward iterates of order $m$ of 0.5 is part of the attractor because all symbols $S_{i}$ have the same history. If one considers a long repetitive subsequence $A$ and reads it backwards then one gets the information for which backward iterate exists: The first symbol marks the first backward iterate of $0.5: 1 \rightarrow$ right, the second

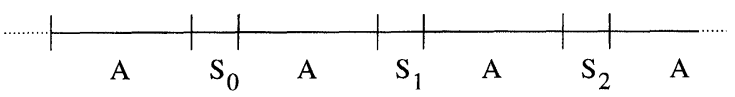

FIGURE 1 Composition of the Feigenbaum sequence. 

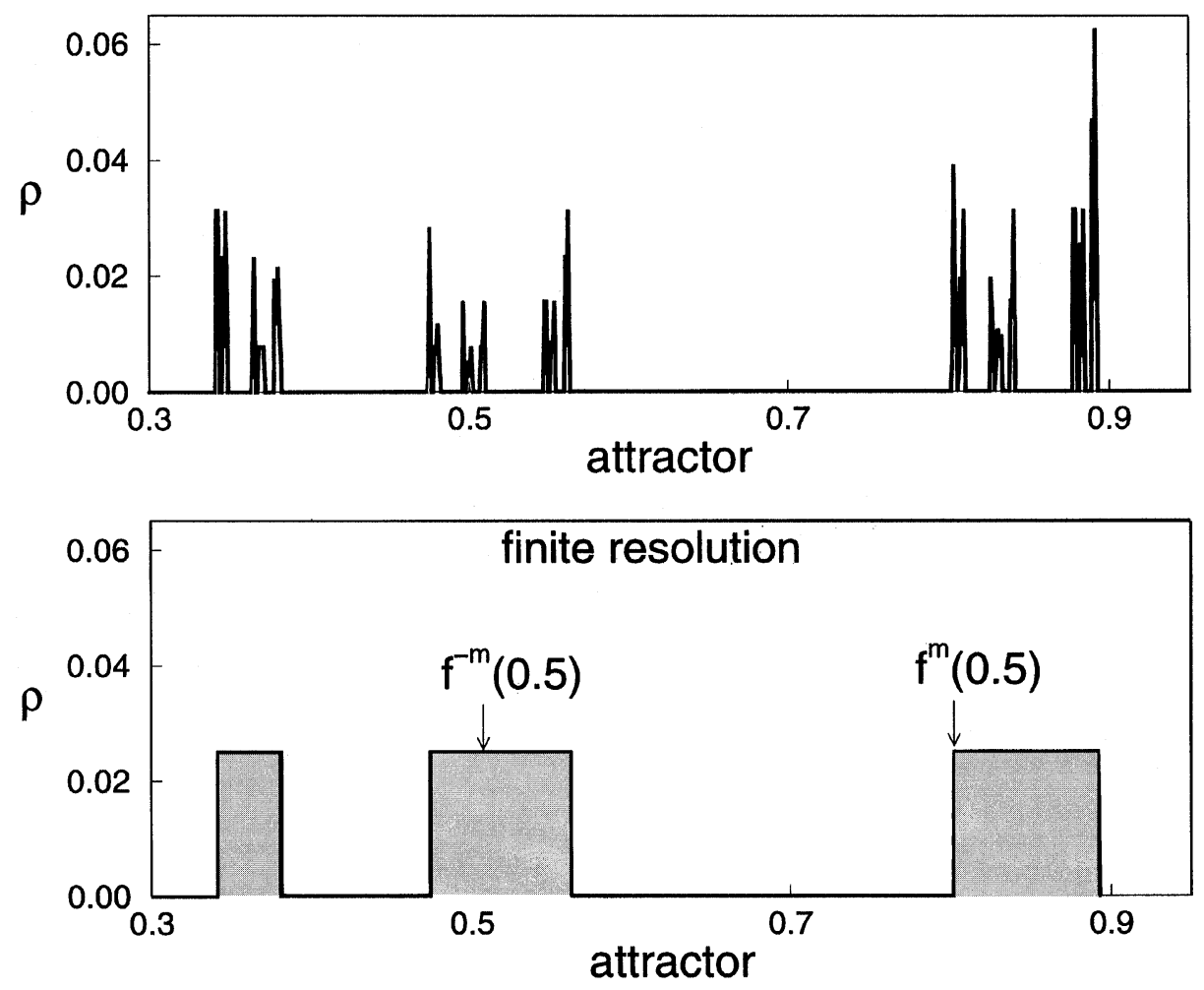

FIGURE 2 Feigenbaum accumulation point: histogram.

symbol marks the second backward iterate of 0.5: $0 \rightarrow$ left, etc. It follows that the rules, which determine whether a point lies left or right of 0.5 , also apply to the first, second, etc., backward iterates of 0.5 . If one chooses a binary partition at a backward iterate of 0.5 , which is part of the attractor, then the symbol sequence generator will be the same (the initial values may be changed) and the resulting symbol sequences are selfsimilar.

Due to the Cantor structure of the attractor the support of the invariant measure consists of a fractal set of points. Looking at this set with a finite resolution one can detect a set of intervals which hint at the hidden selfsimilar structure (Fig. 2). The backward iterates of 0.5 never can match the edges of the tiny intervals since considering a small section around a backward iterate one will always see the microscopic trajectory on both sides of the backward iterate. Consequently the forward iterates of 0.5 match the edges of the tiny intervals. All backward iterates of 0.5 are placed between two forward iterates.

The partition at a forward iterate or at a point which is not part of the attractor leads to a periodic sequence. Considering a small section around the splitting point one will see the microscopic trajectory only on one (forward iterate) or on no (point is not part of the attractor) side of the splitting point. The symbols $S_{i}$ are identical. The symbol sequence is periodic. One finds selfsimilarity on a finite number of scales only.

\section{ENTROPY, COMPLEXITY AND PREDICTABILITY}

Now we will apply Shannon's entropy concept to the analysis of the sequences discussed above. Let us 
start with several definitions [7-9]: We assume that

$$
C_{1}, \ldots, C_{\lambda}
$$

is the alphabet of the sequence which consists of $\lambda$ different letters. A definite subsequence (a block) of length $n$ is denoted by

$$
\begin{gathered}
A_{1}, \ldots, A_{n} \quad \text { where } A_{i} \in\left\{C_{1}, \ldots, C_{\lambda}\right\} \\
\text { for all } i=1,2, \ldots, n .
\end{gathered}
$$

Further we assume that

$$
p^{(n)}\left(A_{1}, \ldots, A_{n}\right)
$$

is the probability of the subsequence $A_{1}, \ldots, A_{n}$ and that

$$
p\left(A_{n+1} \mid A_{1}, \ldots, A_{n}\right)
$$

is the probability to find after the block $A_{1}, \ldots, A_{n}$ the letter $A_{n+1}$. With these notations we define:

1. The entropy of a block of length $n$ :

$$
H_{n}:=-\sum_{A_{1}, \ldots, A_{n}} p^{(n)}\left(A_{1}, \ldots, A_{n}\right) \log p^{(n)}\left(A_{1}, \ldots, A_{n}\right) .
$$

2. The conditional entropy as the mean uncertainty of the letter which follows a block of length $n$ :

$$
\begin{aligned}
& h_{n}:=H_{n+1}-H_{n}, \\
& h_{0}:=H_{1} .
\end{aligned}
$$

3. The entropy of the source (after Shannon, Khinchin und McMillan)

$$
h:=\lim _{n \rightarrow \infty} H(n)=\lim _{n \rightarrow \infty} h_{n} .
$$

For Bernoulli-processes we have

$$
h_{0}=h_{1}=h_{2}=\cdots=h=\log \lambda \text {. }
$$

For first order Markov-processes we get

$$
h_{0}>h_{1}=h_{2}=\cdots=h \text {. }
$$

For processes with the period $p$ we find

$$
H_{n}=\text { const for all } n \geq p
$$

and

$$
h_{n}=0 \text { for all } n \geq p \text {. }
$$

For quasiperiodic processes holds

$$
h_{n} \sim n^{-1} \text { for } n \rightarrow \infty \text {. }
$$

Evidently the sequences generated by the logistic map at the Feigenbaum point, which correspond to a limit of multiperiodicity, belong to this class. As shown in our earlier work [4-6] the entropies of these sequences behave asymptotically as

$$
\begin{gathered}
H_{n}=(\log n)+\log 1.5 ; \\
h_{n}=\frac{4}{3 n} .
\end{gathered}
$$

The same asymptotics have the rabbit sequences [11]. In some sense quasiperiodic processes are the most complex structures among the processes discussed above. Lai and Grebogi [14] characterize a complex structure by the properties:

- the system consists of many components,

- the components can be either regular or irregular, - the components exist on different length and/or time scales,

- the system exhibits a hierarchy of structures.

We have discussed above several characteristics of the hierarchy of structures shown by the Feigenbaum sequences. We believe that the Feigenbaum sequence may be considered as the prototype of a complex structure. At least for linear structures the conditional entropy and the way this function is decaying is a powerful measure for the hierarchy of structures contained in the system. Some time ago Grassberger [3] introduced the so-called effective measure complexity (EMC),

$$
\mathrm{EMC}=\sum_{0, \ldots, \infty}\left(h_{n}-h\right)
$$


The EMC is zero for Bernoulli-sequences and has a finite value for Markov-processes and for periodic processes. However for our particular example the EMC is divergent. Therefore we propose to consider in this case the function

$$
g_{n}=h_{n}-h
$$

and call $g_{n}$ the "function of measure complexity" (FMC). The FMC allows the classification of sequences with infinite EMC. The FMC expresses the character of the memory of the process and the way the correlations are decaying. In this connection we remember that already Szepfalusy [13] introduced in 1989 several classes of sequence structures corresponding to the way of decay of the FMC. Following his ideas we introduce the following classes:

- sequences with divergent EMC and an FMC which decays at $n \rightarrow \infty$ according to the power law

$$
g_{n} \sim \frac{g}{n^{\alpha}}
$$

with $\alpha<1$;

- sequences with divergent EMC corresponding to the limit $\alpha=1$ of the class one;

- sequences with power law decay and $\alpha>1$;

- periodic sequences, corresponding to the case that the limit is reached in a finite number of steps $g_{n}=h_{n}=0$ for $n \geq n_{\max }$ where $n_{\max }=$ finite;

- sequences with Markov character corresponding to a $g_{n}$ which reaches the limit after a finite number of steps or by an exponential decay of $g_{n}$. In this case the limit $h$ is assumed to be finite;

- sequences with Bernoulli character with $h=1$ and $g_{n}=0$ for all $n$.

We propose here to consider all sequences belonging to the first two classes as complex linear structures by definition. The third class is clearly distinct from the first two classes, eventually we may denote it as semi-complex. The complex structures in the sense defined above are characterized by one index $\alpha$. In this sense the Feigenbaum sequences as well as the rabbit sequences belong to the complexity class $\alpha=1$.

A still more refined classification is possible by a scaling formula proposed by Ebeling and Nicolis [4],

$$
H_{n}=n h+g n^{\mu_{0}}(\log n)^{\mu_{1}}+e
$$

with

$$
0 \leq \mu_{0}<1 \quad \text { or } \quad \mu_{0}=1, \mu_{1}<0 .
$$

This leads to the following asymptotics of the FMC:

$$
g_{n}=\frac{g}{n^{1-\mu_{0}}} \cdot\left(\mu_{0}(\log n)^{\mu_{1}}+\mu_{1}(\log n)^{\mu_{1}-1}\right) .
$$

In the special case $\mu_{1}=0$ and $\mu_{0}=1-\alpha$ we come back to the previous classification by one index. The Feigenbaum sequence corresponds to $\mu_{0}=0$ and $\mu_{1}=1$.

In all cases where $g \neq 0$ holds, we find long-range order in the sequence [9]. We consider long-range order as a necessary property of complexity. In this way we may define, generalizing the investigated properties of the Feigenbaum sequence: A linear symbol sequence is called "complex sequence" if, in the limit of large $n$, the FMC $g_{n}=h_{n}-h$ decays according to a power law with $\alpha \leq 1$. In this case the sequence is characterized by an infinite hierarchy of structures. A finer classification is possible by means of the "index of complexity" $\alpha$ or by the two "indices of complexity" $\mu_{0}=1-\alpha$ and $\mu_{1}$ according to the scaling given above [4].

Let us consider now the question of predictability of complex sequences. Due to the existence of a hierarchy of correlations the predictability of complex sequences and in particular the local predictability may be rather high [9]. We define the average predictability as the difference between the maximal uncertainty and the average uncertainty: In $\log (\lambda)$-units the average predictability of the state following a trajectory (history) of length $n$ may be expressed by

$$
r_{n}=1-h_{n}
$$


This is a number between 0 and 1 , where 0 corresponds to Bernoulli processes and 1 e.g. to periodic or stable deterministic processes. Formally we may express the predictability as an average

$$
r_{n}=\left\langle r_{n}\left(A_{1} \cdots A_{n}\right)\right\rangle=1-\left\langle h_{n}\left(A_{1} \cdots A_{n}\right)\right\rangle .
$$

This leads us to the definition of a local uncertainty of the predictions of the state following after the particular subtrajectory $A_{1} \cdots A_{n}$ of length $n$ by the expression

$$
\begin{aligned}
r_{n}\left(A_{1} \cdots A_{n}\right)=1-\sum & p\left(A_{n+1} \mid A_{1} \cdots A_{n}\right) \\
& \times \log p\left(A_{n+1} \mid A_{1} \cdots A_{n}\right)^{-1} .
\end{aligned}
$$

The second term expresses the conditional uncertainty of the next state (1 step into the future) following behind the measured trajectory $A_{1} \cdots A_{n}$ $\left(A_{i} \in\right.$ alphabet $)$. The predictability $r_{n}\left(A_{1} \cdots A_{n}\right)$ is a quantity which is local and fluctuates if we go along the string, since the local "history" $A_{1} \cdots A_{n}$ changes from position to position. For practical applications, one is very much interested in such local predictabilities. The reason is that one needs in practical applications always concrete predictions based on observations of some concrete history of finite length $n$. In other words one is more interested in concrete predictions than in somehow abstract "average predictabilities". The Feigenbaum sequence is a very good example how one can predict the local continuation of the string if some prehistory is known. One can make good predictions if, locally, one of the known grammatical rules apply. Just one example of a predictability with certainty 1 : If we observe a letter " 0 " in the string, we are $100 \%$ sure that the next letter will be a " 1 ", the predictability is one $r_{1}(" 0 ")=1$. The continuation of a " 0 " by a " 1 " is certain since the grammatical rules do not allow to find the subsequence " 00 ". If we observe a " 1 " in the string we are rather uncertain, whether the next letter will be a "1" or a " 0 ", however we can reach more and more certainty of the prediction by looking at the prehistory of the observed " 1 ". By comparing the local prehistory with the known grammatical rules we are able to make sometimes very good predictions. In this way the Feigenbaum sequence may serve as an excellent example showing how the study of grammatical rules may serve to solve the problem of local predictions.

In earlier work we considered entropies and local predictabilities in texts and in biosequences on a more empirical basis [8,9] and by developing theoretical models [15]. We have found some evidence that for texts we have $\alpha<1$ and $1 /(1-\alpha)=$ integer. On the basis of several examples we could show that the predictability in texts and in biosequences fluctuates indeed rather strongly and may locally be very good. For example, after a comma or a point in a text must come a space and we are sure about this up to possible printing errors. This of course is due to grammatical rules which the letters in a text have to observe. Much less is known about the grammatical rules in biosequences [16]. We note that the local structures in strings may also be considered from the point of view of correlated fluctuations [17-19]. Another closely related fluctuation quantity is the transinformation [20]. For DNA-strings extensive calculations of the transinformation are available [20].

\section{CONCLUSIONS}

We have given in this work a more detailed analysis of the structure of the sequences generated by the logistic map at the accumulation point. In some sense these sequences are a prototype of complex linear structures. Coming from parameters below the Feigenbaum point, the sequences show multiperiodicity and with decreasing distance to the accumulation point, more and more complicated structures arise. The structures at the accumulation point itself are hierachical and may be characterized by grammatical rules in the way shown in Sections 2 and 3 of this work. Another way to express the hierarchy of structures is by the way how the conditional entropies decay. Infinite hierarchies of structures correspond to power law tails in the decay of the conditional entropies with $n \rightarrow \infty$. Based on the characteristic exponents of the 
entropy decay, the "degree of complexity" of a given infinite sequence may be characterized by one index $\alpha \leq 1$ or by a set of indices. Finally we have shown that complex sequences allow sometimes rather good local predictions of the continuation of the string, if locally grammatical rules may be applied. In this way we have shown that complexity and predictability do not exclude each other. Complex structures may be predicted if the hierarchical order structures may be detected. Thus as a rule good predictions of complex structures require extended research on grammatical rules or local histories.

\section{References}

[1] Feigenbaum, M.J. (1978). Quantitative universality for a class of nonlinear transformations, J. Stat. Phys. 19, 25-52.

[2] Schuster, H.G. (1989). Deterministic Chaos: An Introduction. VCH Verlag, Weinheim.

[3] Grassberger, P. (1986). Toward a quantitative theory of self-generated complexity, Intern. Journ. Theo. Phys. 25, 907-938.

[4] Ebeling, W. and Nicolis, G. (1992). Word frequency and entropy of symbolic sequences: a dynamical perspective, Chaos, Solitons \& Fractals 2, 635-650.

[5] Ebeling, W., Freund, J. and Rateitschak, K. (1996). Entropy and extended memory of discrete chaotic dynamics, Int. J. Bifurc. Chaos 6, 611 .

[6] Rateitschak, K., Freund, J. and Ebeling, W. (1996). Entropy of sequences generated by nonlinear processes: the logistic map. In: Entropy and Entropy Generation: Fundamentals and Applications, ed. J. S. Shiner, Kluwer, Dordrecht.

[7] Freund, J., Ebeling, W. and Rateitschak, K. (1996). Selfsimilar sequences and scaling of dynamical entropies, Phys. Rev. E 54, 5561.
[8] Ebeling, W., Pöschel, T. and Albrecht, K.-F. (1995) Entropy, transinformation and word distribution of information-carrying sequences, Int. J. Bifurcation \& Chaos $\mathbf{5}$, 51-61.

[9] Ebeling, W. (1997). Dynamic entropy and predictability of sequences with LRO, Physica D 109, 42-52.

[10] Hopcroft, J.E. and Ullman, J.D. (1979). Introduction to Automata Theory, Languages and Computation. AddisonWesley.

[11] Gramss, T. (1995). Entropy of the symbolic sequences for the critical circle map, Phys. Rev. E 50, 2616-2620.

[12] Schroeder, M.R. (1991). Fractals, Chaos, Power Laws. Freeman, New York.

[13] Szepfalusy, P. (1989). Characterization of chaos and complexity by properties of dynamical entropies, Physica Scripta T 25, 226-326.

[14] Lai, Y. and Grebogi, C. (1996). Complexity in hamiltondriven dissipative chaotic dynamical systems, Phys. Rev. E 54, 4467-4477.

[15] Rateitschak, K., Ebeling, W. and Freund, J. (1996). A nonlinear dynamical model for texts, Europhys. Lett. 45, 401.

[16] Ebeling, W. and Frömmel, C. (1998). Entropy and predictability of information carriers, BioSystems, in press.

[17] Stanley, H.E. et al. (1994). Statistical mechanics in biology: how ubiquitous are long-range correlations? Physica A 205, 214.

[18] Ebeling, W. and Neiman, A. (1995). Long-range correlations between letters and sentences in texts, Physica A 215, 233.

[19] Ebeling, W., Neiman, A. and Poeschel, T. (1995). Dynamic entropies, long-range correlations and fluctuations in complex linear structures. In: Coherent Approach to Fluctuations (Proc. Hayashibara Forum 1995). World Scientific, Singapore.

[20] Herzel, H. and Grosse, I. (1995). Measuring correlations in symbol sequences, Physica A 216, 518-542; Correlations in DNA-sequences, Phys. Rev. E 55, 800. 


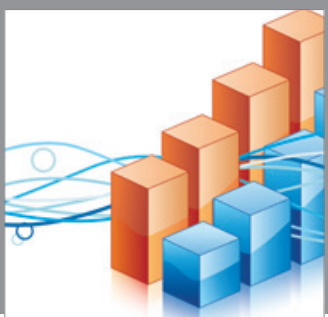

Advances in

Operations Research

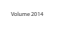

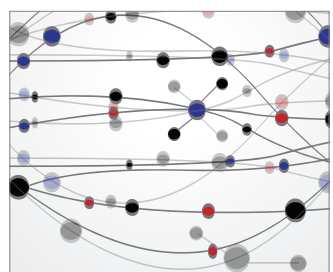

\section{The Scientific} World Journal
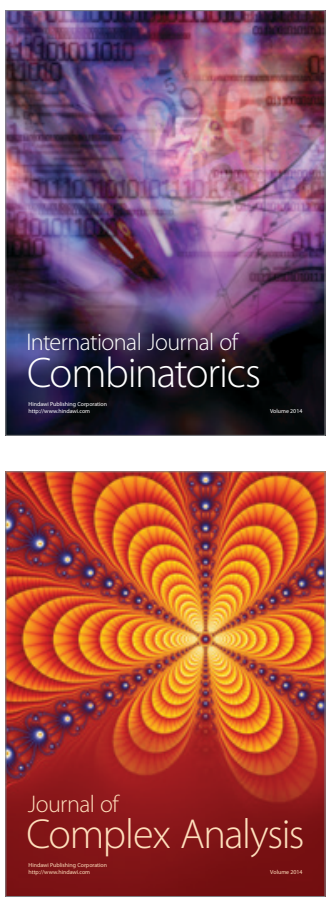

International Journal of

Mathematics and

Mathematical

Sciences
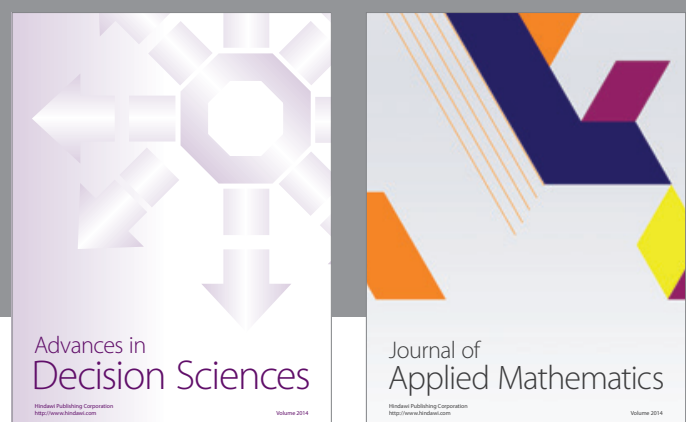

Journal of

Applied Mathematics
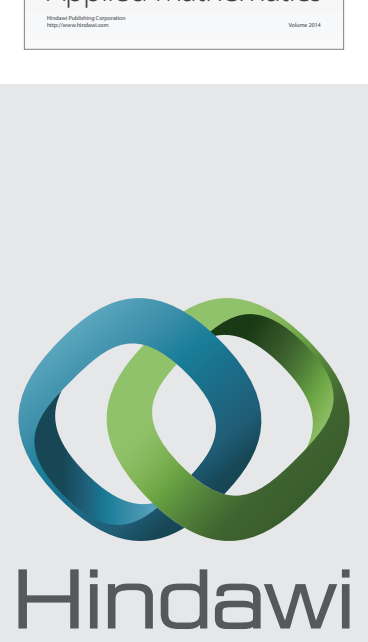

Submit your manuscripts at http://www.hindawi.com
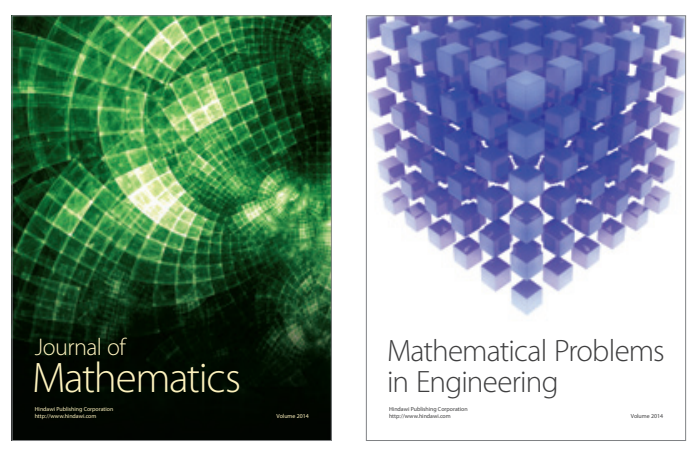

Mathematical Problems in Engineering
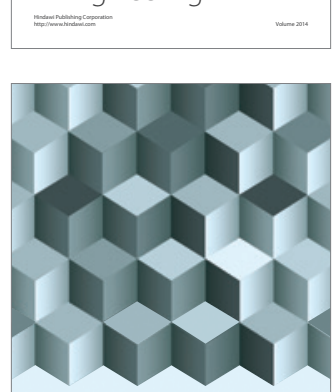

Journal of

Function Spaces
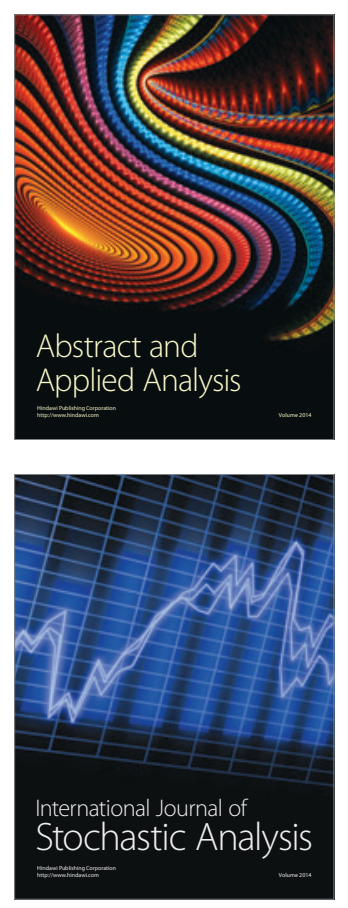

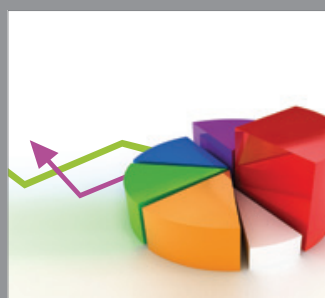

ournal of

Probability and Statistics

Promensencen
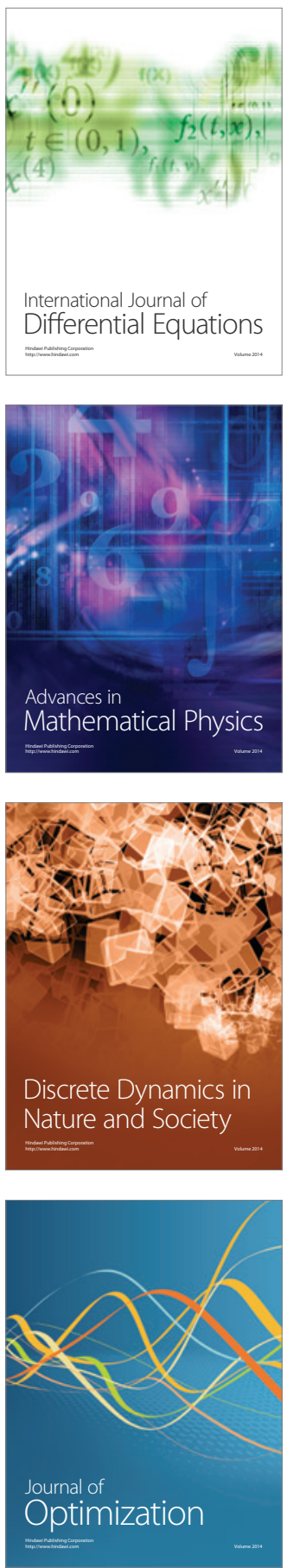